\title{
Magnetic Field Effects of Double-Walled Carbon Nanotubes
}

\author{
A. Latgé ${ }^{1}$, D. Grimm ${ }^{1,2}$, and M. S. Ferreira ${ }^{3}$ \\ ${ }^{1}$ Instituto de Física, Universidade Federal Fluminense, 24210-340, Niterói, RJ, Brazil \\ ${ }^{2}$ IFW Dresden, P.O. Box 270116, D-01171 Dresden, Germany \\ ${ }^{3}$ Physics Department, Trinity College Dublin, Dublin 2, Ireland
}

Received on 8 December, 2005

\begin{abstract}
A theoretical discussion of electronic and transport properties of a particular family of double-wall carbon nanotubes, named commensurate structures of the armchair type $(n, n) @(2 n, 2 n)$ is addressed. A single $\pi$-band tight binding hamiltonian is considered and the magnetic field is theoretically described by following the Peierls approximation into the hopping energies. Our emphasis is put on investigating the main effects of the geometrical aspects and relative positions of the tubes on the local density of states and on the conductance of the system. By considering intershell interactions between a set of neighboring atoms on the walls of the inner and outer tubes, we study the possibility of founding Aharonov-Bohm effects in the DWCNs when a magnetic field is applied along the axial direction.
\end{abstract}

Keywords: Carbon nanotubes; Conductance; Magnetic field

\section{INTRODUCTION}

Double-wall carbon nanotubes (DWCNs) are coaxial two tube-systems which have attracted a great deal of interest because of their promising mechanical and electronic properties as well as potential applications. They may be achieved, for instance, by peapod -derived methods[1] or synthesized by pulsed arc discharge processes and low pressure catalytic chemical vapor deposition[2], among others. The distance between both the cylinders are quite the same as found between carbon planes in graphite structures, and therefore the correspondent intertube electronic interactions have a vander-Waals like energy scale which is much inferior than the intra-atomic correlation within each single tube. X-ray diffraction analysis of the structural transformation from singlewall carbon nanotubes to DWNTs via C-60 peapods[3] determined the intertube spacing between inner and outer tubes as $0.36 \pm 0.01 \mathrm{~nm}$, and that the tubes are loosely coupled to each other. Interlayer potential energies for different chirality pairs of tubes composing the double-walled system have been calculated[4-6] adopting the van-der-Waals potential.

If the ratio between the two unit cell lengths along the common tube axis is a rational number the DWCN is called a commensurate tube system [examples are the $(n, n) @(2 n, 2 n)$, and $(\mathrm{m}, 0) @(2 \mathrm{~m}, 0)]$. Previous theoretical works on DWCNs[7, 8] have discussed the stability of the electronic structure of the coupled system based on symmetry arguments and the necessity of introducing relative positions between the inner and outer tubes to induce opening of an energy gap. This is in contrast to the electronic structure of identical metallic nanotubes arranged close packed in bundles which exhibit a pseudogap at the Fermi level[9]. Aperture of energy gaps in CNs may be achieved via the application of a magnetic field, too, leading also to the well known Aharonov-Bohm phenomena.

Considering that single-walled $\mathrm{CNs}$ have been proven to be essentially ballistic[10] it should be interesting to investigate the conductance of DWCNs. By submerging DWCNs into liquid mercury, Kajiura et al.[11] were able to measure the conductance dependence on the length of the structures. They concluded that the electronic transport in the double-walled systems is quasi-ballistic like at room temperature, and that the mean free path is of the order of the length of the tubes. Here we investigate how the conductance of a commensurate DWCN is affected by the existence of the interactions between the double walls and how it depends on the relative position of the atoms in the two tubes. Finally we calculate also the conductance in the presence of a magnetic flux and discuss its consequence on the quantum interference phenomena.

\section{THEORY AND RESULTS}

A single $\pi$-band tight binding hamiltonian is used to describe the structure composed of two single nanotubes which is schematically displayed in Figure 1, a short cut of a $(5,5) @(10,10)$ DWCN. It is written as

$$
H=\gamma_{o} \sum_{i, j, t}\left(c_{i, t}^{+} c_{j, t}+c_{j, t}^{+} c_{i, t}\right)+\sum_{i, j} \gamma_{1}\left(r_{i j}\right)\left(c_{i, 1}^{+} c_{j, 2}+c_{j, 2}^{+} c_{i, 1}\right)
$$

where the indices $\mathrm{i}$ and $\mathrm{j}$ define the atomic positions, $r_{i j}=$ $\left|\vec{r}_{i}-\vec{r}_{j}\right|$ the atomic distance and $\mathrm{t}=1,2$ labels the two tubes. The intrawall hopping energy between first neighbors $\gamma_{o}$ is considered of the order of $-2.7 \mathrm{eV}$, while $\gamma_{1}(r)$ is used for the intershell coupling which in principle depends on the distance between the two carbon atoms displayed on the inner and outer tube walls. As mentioned before the interlayer interaction between adjacent layers is sufficiently small compared to the intralayers $\mathrm{C}-\mathrm{C}$ bonding. Different calculations for the potential barrier between the two shells of DWCNTs have been presented, most of them using local density approximation. Here we consider $\gamma_{1}(r)$ as a parameter of our tight binding approximation and use, in a first approach, a cut off scheme to determine the number of atomic interactions to be included. In that direction, a unit cell of four times the achiral nanotube unit cell, as shown in Figure 1, is sufficient to include all interactions within a range varying typically $10 \%$ the value of $\gamma_{1}^{M}$ (maximum intershell hopping energy value, used for a distant pair corresponding to the minimum distance 
between the inner and outer atoms in the DWCN). We consider that the energy intensity reduces accordingly to a potential law.

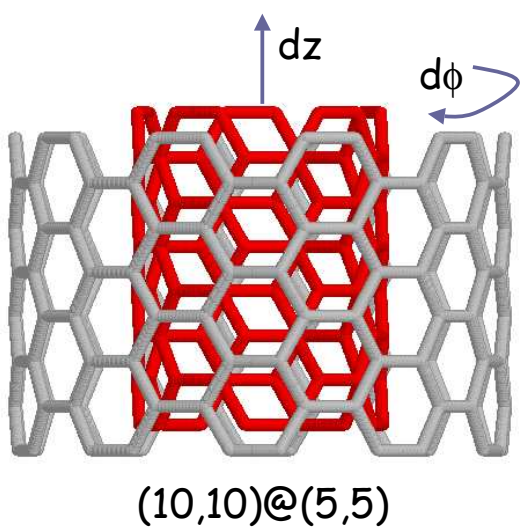

FIG. 1: Schematic view of a $(5,5) @(10,10) D W C N$, showing the relative displacement between the intertube atomic positions along the axial direction $(\Delta z)$ and in the azimuthal angle $(\Delta \phi)$.

A standard real-space renormalization technique is adopted to calculate the system's Green functions[12]. A realistic ingredient to take into account is the orientational disorder between the tubes forming the DWCN structure. Therefore, a relative displacement between inner and outer tubes is allowed, $\Delta z$, as well as an azimuthal rotation, $\Delta \phi$, as indicated in Fig. 1. Both are given in units of percentages of a hexagon. To study the transport properties we use the Landauer formula for the conductance following a Green function formalism[13].

A typical result for the conductance of a DWCN using $\gamma_{1}^{M}$ $=240 \mathrm{meV}$ is presented in Fig. 2(a). Values around $300 \mathrm{meV}$ have been used by Saito et al.[7]. A graphitic like stacking is employed where an inner tube atom lies in the center of a hexagon of the outer tube $(\Delta z=\Delta \phi=50 \%)$. For energies away from the Fermi level, there is a clear dependence of the conductance results on the intensity of the intertube interaction although the conductance suppressions are mainly strong at the band edges. Interesting tight oscillations on the conductance around the Fermi level may also be observed. The influence of the potential depth on the mentioned oscillations near the Fermi level is shown in Fig. 2(b)\&(c) for $\gamma_{1}^{M}=5,20$ and $75 \mathrm{meV}$. A pseudogap in the LDOS in Fig. 2(c) opens reflecting the reduction of two transport channels to only one in the conductance results in Fig. 2(b). Beside the spikes superposing the constant metallic LDOS plateau, we found a constant spectral weight (constant integral) of the LDOS for all the $\varepsilon$ values used in the calculation. Augmenting the potential strength, the gaps also increase in size, are dislocated to higher energies and present more complex oscillation patterns, as shown by the multiple oscillations for $\gamma_{1}^{M}=75 \mathrm{meV}$ in Fig. 2. One should mention that although the conductance suppressions exhibit a significant dependence on the intensity of the interlayer energies, the gaps are extremely narrow for low values of $\gamma_{1}^{M}$.

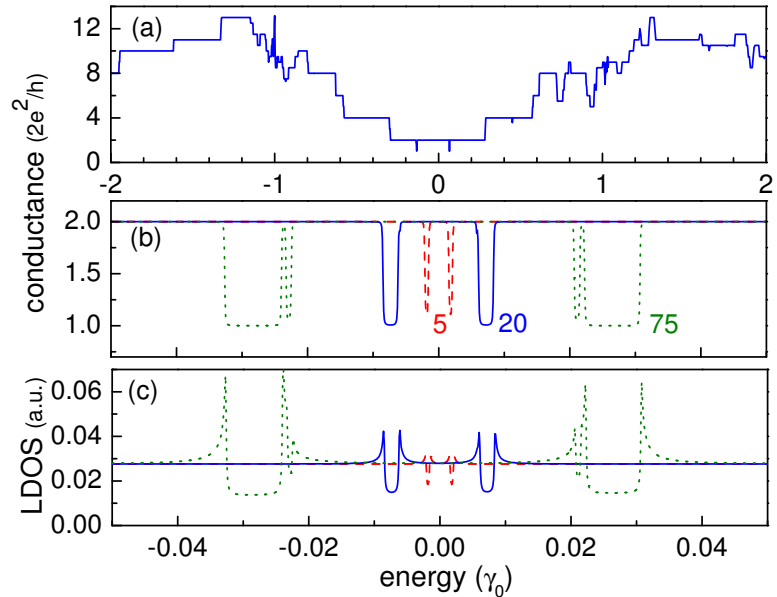

FIG. 2: (a) Conductance of a $(5,5) @(10,10)$ DWCN with graphitic like stacking $\Delta z=50 \%, \Delta \phi=50 \%$ and $\gamma_{1}=240 \mathrm{meV}$. Dependence of the conductance (b) and LDOS (c) of the same DWCN ( $\Delta z=$ $50 \%, \Delta \phi=10 \%$ ) with the intrashell interaction. Dashed red curve corresponds to $\gamma_{1}^{M}=5 \mathrm{meV}$, solid blue to $\gamma_{1}^{M}=20 \mathrm{meV}$, and dotted green to $\gamma_{1}^{M}=75 \mathrm{meV}$.

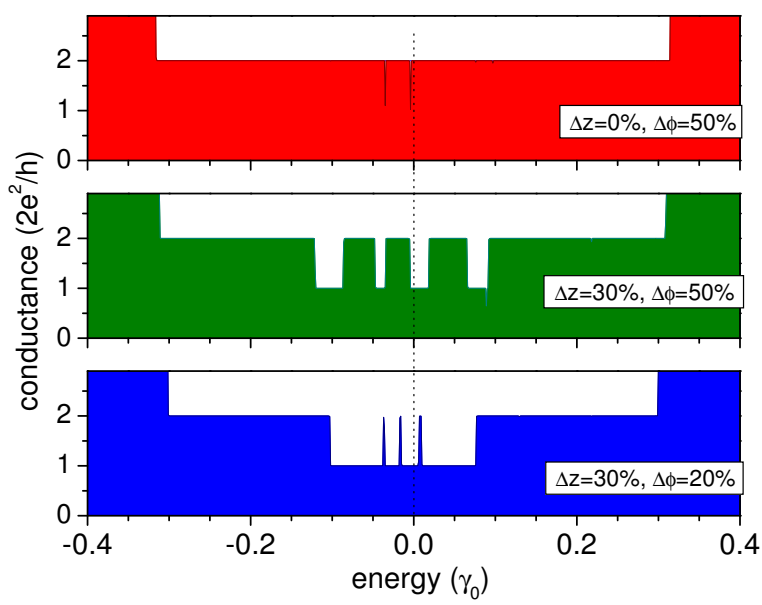

FIG. 3: Conductance of a $(5,5) @(10,10)$ DW with varying inner tube shift $\Delta z$ and rotation $\Delta \phi$. The Fermi level is shown by the vertical dotted line.

Simulations indicate, that the energy barriers for dislocations and rotations of the inner tube are relatively low, allowing a quite easy gliding of the two tubes respective to each other[14, 15]. Therefore, allowing the inner tube to slide and rotate relatively to the outer tube, a reduction of the transport channels or not at the Fermi level occur. In Fig. 3 some conduction calculation results for different values of the inner tube positions are presented for a $(5,5) @(10,10)$ DW. For a better visualization, the Fermi level is indicated by the vertical dotted line and a quite high potential strength of $\gamma_{1}^{M}=240 \mathrm{meV}$ is used. For $\Delta z=0 \%, \Delta \phi=50 \%$ the two transport channels of a pristine tube are obtained, interrupted by 
only two very sharp dips, away from the Fermi level. Slightly shifting the inner tube leads to a completely different pattern; for $\Delta z=30 \%, \Delta \phi=50 \%$ a square saw pattern can be observed and one of the saw is situated at the Fermi level, causing a transport reduction to one channel. A small rotation around the inner tube axis leads to a broad conduction reduction around the Fermi level, with only few peaks superposed preserving the two channels, clearly visible for $\Delta \phi=20 \%$ in Fig. 3. This indicates unambiguously the important role played by the small variations of the assembly of the two tubes on the transport properties of the system, resulting in symmetry changes and broad transport reduction regions, even in the presence of merely weak intertube atomic interactions like the one used in the present calculations.

Alternatively, a simpler intra-tube interaction picture was adopted in which each atom laying in the inner tube interacts with three first neighbors on the outer tube, with $\gamma_{1}=\gamma_{o} / 8$ for the closer two atoms and $\gamma_{o} / 10$ for the other two next intertube neighbors. The results of the local density of states (LDOS) for the studied DWCNs, using such a naive calculation, have provided to be similar to the sum of the two individual tube LDOS. For the conductance, however, we found changes on the conductance as compared to the isolated SWCNs case, mainly at those energies at which new quasi 1-dimensional bands appear, corresponding to the positions of new steps in the conductance.
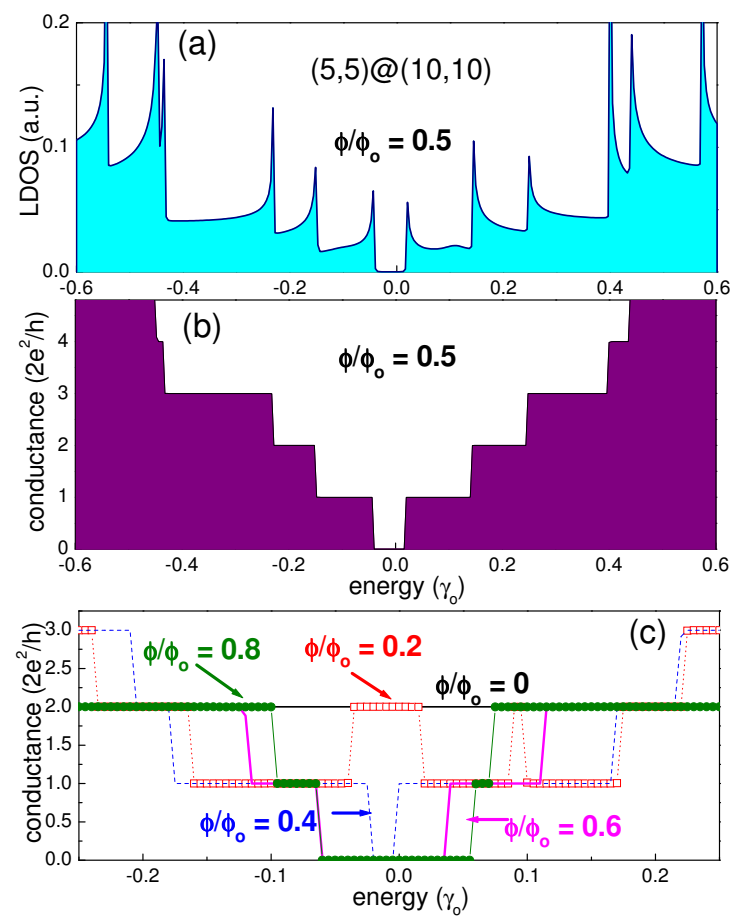

FIG. 4: DWCN $(5,5) @(10,10):$ (a) LDOS and (b) Conductance at a magnetic flux (relative to the outer tube) equal to $\phi / \phi_{o}=0.5$; (c) Conductance for different values of magnetic flux.

To consider the effects of a magnetic field threading the DWCN along the axial direction we adopted the Peierls approximation[17]. In this theoretical picture, phase factors $\Delta G_{\mathbf{R}, \mathbf{R}^{\prime}}$ are added to the hopping energies, depending on the local atomic neighborhood determined by the vector $\mathbf{R}$ and $\mathbf{R}^{\prime}$ and the intensity of the magnetic flux $\phi$, which is written in terms of the quantum flux $\phi_{o}=h / e=4.13 \times 10^{-15} \mathrm{Tm}^{2}$. The matrix elements of the hamiltonian may be written as

$$
H_{i, j}=\gamma e^{i e / \hbar \Delta G_{\mathbf{R}, \mathbf{R}^{\prime}}}
$$

One should notice that the flux within each one of the tubes composing the DWCN is not the same: in the studied commensurate system, the internal flux is one fourth the external one. Results for the LDOS and conductance of a

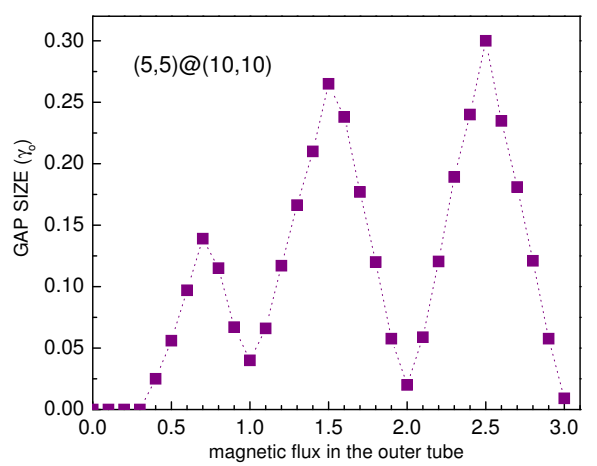

FIG. 5: Gap size dependence on the magnetic flux (outer tube) for the $(5,5) @(10,10)$ DWCN.

$(5,5) @(10,10)$ DWCN are shown in Figure 4(a) and (b), respectively, using the simple electronic model and considering a magnetic flux of 0.5. When the magnetic field is turned on, the number of electronic channels next to the (zero) Fermi energy is reduced at particular energy windows, eventually even leading to the suppression of the conductance. This is accompanied by the energy gap aperture in the LDOS, as it is illustrated for the special case of half a magnetic flux quantum $\phi / \phi_{o}=0.5$ in Fig. 4(b) and for different flux values in Fig. 4(c). To highlight the effect of the magnetic field on the conductance of the system, we show in Fig. 5 the dependence of the gap size on the intensity of the flux going through the external tube. For a pristine SWCN the gap size oscillates (Aharonov Bohm manifestation) with a period equal to one quantum flux. In the present results one clearly notices that the periodicity on the magnetic flux, is not fully achieved for the studied DWCN: the gap retains null up to a magnetic flux (external tube) of the order of $0.35 \phi_{o}$ and as the magnetic field increases it oscillates with the $\phi_{o}$ periodicity but does not attains the same values. This may be interpreted as being a consequence of the competition between the intertube interaction energies and the confinement induced by the magnetic field. More work in this direction is needed to understand the gap size evolution with the external field. 


\section{CONCLUSIONS}

We have presented a theoretical discussion of physical properties of double walled carbon nanotubes. Emphasis was put on the analysis of the effects of geometrical arrangements and the relative position between outer and inner atoms on the electronic transport. Our results clearly indicate the aperture of pseudogaps in the LDOS followed by conductance reductions next to the band center (Fermi level) due to small configurational changes. Similar results in the density of states near the Fermi level of $(5,5) @(10,10)$ DWCNs have been previously reported [8] using parametrized tight-binding techniques with parameter determined by ab initio calculations. The different atomic positions of the two tubes eventually reduces the symmetries of the system, raising some degeneracy and leading to important electronic-like transitions. These effects are equivalent to the ones achieved when the DWCN is considered under the action of magnetic fields, as described above. One should mention, however, that the quantum phenomena described in the manuscript, like gap opening and conductance reduction may be viewed only qualitatively since the details of the results, such as the energy positions of the conductance changes and gap sizes, do depend on the intensity of the hopping energy parameters. More elaborate theories, based on first principles, are necessary to provide them in different geometrical configurations.

\section{Acknowledgments}

We thanks the partial financial support of CAPES (PROBRAL), CNPq and grant of Millenium. A. L. thanks the hospitability of the Leibniz Institute where part of this work was done and D.G. acknowledges the DFG PI 440/3.
[1] S. Bandow, M. Takizawa, K. Hirahara, M. Yudasaka, and S. Iijima, Chem. Phys. Lett. 337, 48 (2001).

[2] A. Grüneis, M. H. Rummeli, C. Kramberger, A. Barreiro, T. Pichler, R. Pfeiffer, H. Kuzmany, T. Gemming, and B. Bühner, submitted to Carbon (2005).

[3] M. Abe, H. Kataura, H. Kira, T. Kodama, S. Suzuki, Y. Achiba, K. Kato, M. Takata, A. Fujiwara, K. Matsuda, and Y. Maniwa, Phys. Rev. B 68, 41405 (2003).

[4] R. Saito et al. Chem. Phys. Lett. 348, 187 (2001).

[5] S. B. Sinnott, O. A. Shenderova, C. T. White, and D. W. Brenner, Carbon 36, 1 (1998).

[6] J. P. Lu, X.-P. Li, and R. M. Martin, Phys. Rev. Lett. 681551 (1992).

[7] R. Saito, G. Dresselhaus, and M. Dresselhaus, J. Appl. Phys. 73, 494 (1993).
[8] Y.-K. Know and D. Tomanek, Phys. Rev. B 58, R16001 (1998).

[9] Y.-K. Know, S. Saito, and D. Tomanek, Phys. Rev. B 58, R13314 (1998).

[10] C. T. White and T. N. Todorov, Nature 393240 (1998).

[11] H. Kajiura, H. Huang, and A. Bezryadin, Chem. Phys. Lett. 398, 476 (2004).

[12] C. G. Rocha, A. Latgé, and L. Chico, Phys. Rev. B 72, 85419/16 (2005).

[13] M. B. Nardelli, Phys. Rev. B 60, 7828 (1999).

[14] J.-C. Charlier, and J.-P. Michenaud, Phys. Rev. Lett. 70, 1858 (1993).

[15] A.H.R. Palser, Phys. Chem. Chem. Phys. 14459 (1999).

[16] Shi-Dong Liang, Physica B 352, 305 (2004).

[17] J. M. Luttinger, Phys. Rev. 84, 814 (1951). 